\section{Communication Aspects in Health Care Work in Nepal}

\section{Marit Bakke and Madhusudan Subedi}

\begin{abstract}
The aim of this article is to describe the communication aspects in health care work in Nepal. It begins with the idea that communication is important in social development and covers some facts about health conditions and health services in Nepal. It gives a brief introduction to different development paradigms as well as an assessment of their relevance for social change and some basic principles in communication theory. The article then describes communication strategies in which people get a chance to express cultural beliefs with respect to health and treatment practices, and how this knowledge can be applied to facilitate increased use of health services. Cultural conceptions play a crucial role in successful health communication. The article gives one illustration of this by presenting the terms used by mothers in five different ethnic groups in Nepal to describe acute respiratory infections (ARI) and illnesses among children. Implications for health communication are presented at the end of this article.
\end{abstract}

Key Words: Health Communication, Communication theory, Cultural Preconditions, Cultural Strategy, Social Development

\section{Introduction}

Communication is about exchanging ideas, information and knowledge. It is also about transferring new ideas, information and knowledge from people who know to people who do not know. This is done in order to change people's ideas and to increase their knowledge about different issues, often with the intention of also changing people's behaviour. Such communication processes are very important when we are interested in social development which can improve people's social and economic conditions. Right from the beginning of this article, we can state that communication is an important tool for handling social problems, but not all problems are communication problems. Why this is so will be discussed later in this article.

Good health is fundamentally and intrinsically important for living a worthwhile human life and for individuals to participate in the development of a society. This is the case throughout the world, but even more so in developing countries. For instance, as Director-General of the World Health Organization (WHO), Gro Harlem Brundtland (1999: vii) stated that "Remarkable gains in health, rapid economic growth and unprecedented scientific advance - all legacies of the 20th century - could lead us to a new era of human progress." She continued (ibid.: viii):

Opportunity entails responsibility. Working together we have the opportunity to transform lives now debilitated by disease and fear of economic ruin into lives filled with realistic hopes. I have pledged to place health at the core of the global development agenda. That is where it belongs. Wise investments in health can prove to be the most successful strategies to lead people out of poverty.

This is indeed an ambitious vision, particularly when we begin to discuss how it can be implemented in different countries with specific economic, social, physical and cultural conditions. It is often argued that good health services are crucial for improving people's health condition. Research has shown (see for example Justice 1987; Dixit 1999; Subedi 2001) and health workers know from experience, that the presence of health services is not 
sufficient because many people do not use them due to physical/geographic, economic and cultural barriers. The process of exclusion and the existence of cultural conditions join in an alliance that is very unfortunate for certain vulnerable groups. Also access to all other human development resources is a crucial prerequisite for enhancing people's living condition in general, and for improving their health in particular.

Any communication process or strategy must focus on a particular issue or message. In this article the issue is how communication can be used to cope with malnutrition and certain types of illnesses that are prevalent among the Nepalese population. The next section describes some facts about health conditions and health services in Nepal. Section 3 gives a brief introduction to different development paradigms as well as an assessment of their relevance for social change. The article's section 4 describes some basic principles in communication theory. These principles are explored further in section 5. This section includes a presentation of a communication strategy in which people get a chance to express cultural beliefs with respect to health and treatment practices, and how this knowledge can be applied to facilitate increased use of health services. To include indigenous health knowledge has to be considered seriously in such a strategy. Thus, the challenge seems to be to find ways to apply people's beliefs and knowledge as a basis for establishing health services in which knowledge from indigenous health practices as well as from school medicine can be merged. Section 6 illustrates the concept of cultural preconditions by presenting the terms used by mothers in five different ethnic groups in Nepal to describe acute respiratory infections (ARI) and illnesses among children.

\section{Health Conditions and Health Care in Nepal}

Over the last 50 years, considerable gains in health status have been achieved. There has been considerable progress in the coverage of population with the essential elements or programs of health care (Martinez and Koirala 2002; MoH 1999; MoH 2004; HMG/NPC 2002; NESAC 1998). Some efforts have been made by the various governments in Nepal during the successive five years plan to improve the health status of the people and enhance the access to basic health care. After the commencement of multi party system in Nepal in 1990, notable progress has been made in improving the primary health care service network by establishing primary health care centres at the electoral constituency level, subhealth post at the Village Development Committee (VDC) level and improving the outreach services with the provision of female Maternal and Child Health Workers (MCHW), Village Health Workers (VHW) and Female Community Health Workers (FCHW) at the VDC level. There has been some progress in improving access to water supply and sanitation, although great differences still exist between and within social groups. Immunization has shown the most dramatic improvement compared to other health programs.

However, the health status and health services available to the people in rural Nepal are among the worst ever found in the modern world (Woollard, 2005). Health care centers are frequently lacking in trained personnel and medical supplies. A large segment of the population relies on traditional healers. Children are particularly vulnerable, because they are less likely to be taken long distances to health centers, and they are more prone to disease than adults. Pneumonia and diarrhea are the two leading causes of death among the children under five in Nepal. By the end of the ninth five year plan (2001), it was reported that among children under the age of five, mortality rate is 93.6 per 1000 for urban areas, 
147.3 per 1000 in the Terai and 201 per 1000 for those living in the mountain regions of Nepal. In the year 2002, there were a total of 3265 Nepali doctors in the country, out of which 2752 (71\%) were working in a hospital setting and 44 percent of them were working in the Kathmandu Valley alone. The doctors working in the mountain and hill districts constituted 2 percent and 16 percent respectively (Marasini 2003 cited in Woollard 2005). In the Kathmandu Valley, there is a ratio of 1 doctor per 850 people compared to 1 per 30,000 outside the Valley. In remote districts the ratio is 1 per 150,000 . There is a wide gap between the urban and rural areas in educational attainment, health care facilities, transportation, communication, and economic status.

Medical services in Nepal are firmly located in the broader context of the international market in health care. Allopathic medicine has become a profitable commodity, promoted and marketed by multinational pharmaceutical companies, and the access among sick persons to medical services is governed by their ability to pay fees. Since the poorest are among the least healthy, market principles usually fail to match health needs and medical care (Subedi, 2001). Health service has never adequately reached the masses. The health service is poor due to the weak basis in the development of policy and planning, poor commitment during implementation of the health and related programs, disrespect for the health rights of the people, continuing political instability, and the lack of people's participation. There is a need to review the past, admit failures and change policy to allow more participation of the people and their representatives from the villages upward in the decision making processes in health care.

\section{Health Issues as Elements in Social Development}

The description of health conditions in the previous section makes it abundantly clear that we are facing with a serious social problem. This is a problem not only for each individual who suffers from malnutrition and illnesses, but also for Nepal as a country. A healthy population is Nepal's most crucial resource for social and economic development.

The term development can be interpreted in different ways that have implications for the choice of strategy to solve social problems, including health problems. It is common to distinguish between three development paradigms: modernization, dependency, and participatory (Melkote and Steeves, 2001).

The modernization paradigm dominated in academic discussions and practical development work during the 1950s and 1960s. Modernization was particularly seen as something that ought to take place within autonomous Third World countries in order to bring so-called underdeveloped countries into ever higher levels of development. The development was defined as economic growth with rapid and sustained expansion of material production, productivity, and income per head. This would be obtained by introducing capital-intensive, but labour-saving technology that could change the industrial infrastructure. It was also necessary to use communication to bring ideas, knowledge, and skills to people. The communication part was important for creating among people a positive attitude toward modernization, in other words, to create a modernization culture. This required four elements: awareness of the problem of underdevelopment, knowledge about material technology and skills, a positive attitude toward using new technology to cope with underdevelopment, and finally, to change people's behaviour so they actually begin using new technology. These four elements constitute a diffusion process, and it will be described in more detail in the next section. We should also be aware of the fact that centralized planning was seen as the best way to use available resources efficiently on the road 
from under-development toward higher levels of development. This later aspect has significant implications for which parts of the population benefit from development. In Nepal this evidently brings forth the issue of changes in urban and rural areas, in central and remote parts of the country.

The modernization paradigm emphasized the role of mass media for information dissemination in order to obtain technological and social change. High media exposure was believed to make people receptive to change their traditional beliefs and attitudes. The modernization perspective has a tendency to blame individual as well as social constraints for underdevelopment. But it does not recognize that there are external constraints that put limits on development. These external constraints are international trade, economic imperialism of international corporations, and the vulnerability and dependency of recipients of technical assistance programs. Moreover, modernization paradigm failed to differentiate between the developing countries with rich resources and those with low resources. These two types of developing countries may need entirely different development strategies to achieve the best results.

After some years it became apparent that social change in many of the countries that had followed the modernization paradigm did not go in the expected direction. Therefore, in the mid 1970s, there evolved an alternative pathway to development. The dependency paradigm posed the question: why was development not working in some of the developing countries? This paradigm argues that foreign aid, technology, and information have created underdevelopment rather than being a force for development. This relationship implies that capital and technology are introduced from abroad by foreign governments, international organizations such as the World Bank or International Non-Government Organizations
(INGOs) such as the United Nations Development Programme, the World Health Organization, etc. In this way the very same modernization process that should bring countries out of underdevelopment puts them in a state of dependency in an economic, political, and cultural sense. Contracts about trade or for aiding specific projects create economic as well as political dependency. The trans-national corporations and international trade tactics monopolize the economic scene. Foreign aid often implies that developed countries affect conditions on the political and social scene in developing countries (Mishra, 1987). The dependency relationship has also been described as imperialism which reflects a situation in which there are uneven power relations.

Dependency communication theorists analyse mass media as "ideological state apparatuses" which reinforce the dependent character of production relationship. The trans-national corporations penetrate the political sphere via their control over modern technology, and their ideological and economic logic influences the content of education. The advanced capitalist countries sell the most sophisticated communication technologies to less industrialized countries. The myth is being created by these advanced countries that advanced mass media technology could contribute to the process of overcoming some of the problems of underdevelopment. The trans-national corporations control the mass media through news and entertainment programs.

From the 1980s, the participatory paradigm has been more pronounced. The emphasis is put on active participation of the people at the grass roots level, and the process of development is assessed as being specific for each Third World country. This means that there is no universal development strategy, but that a specific one must be designed according to each country's specific natural environments, available natural and human resources, and 
cultural values. Everett Rogers (2000: 127) has defined the participatory paradigm in the following way:

Development is defined as a widely participatory process of social change in a society intended to bring about both social and material advancement (including greater equality, freedom, and other valued qualities) for the majority of people through their gaining greater control over their environment.

The crucial difference between these three ways of looking at development is the degree to which people in a country is taking part in decision making processes regarding social, political, and cultural affairs. In the material modernization approach professional experts and top politicians have made a list of criteria against which the level of development is measured. This is a top-down process. On the other hand, a bottom-up process means that people themselves participate in defining what the important needs are and how they should be met.

Another term that we should know is 'sustainable development'. Melkote and Steeves (2001: 35) writes that this perspective "assumes that maintaining the biological diversity of the planet is essential to the survival of humanity. Hence development that does not prioritize environmental sustainability is doomed to fail.” Subedi (2005: 234) looks at this type of development in a Nepalese context and describes it as process in which desirable social objectives are taken care of. It implies "[...] help for the very poor, marginalized and disadvantaged; self-reliant development; basic health and educational facilities for all; clean water and shelter for all; human beings, in other words, are the resources in this concept." Similarly, Panday's (1999: 4) concern about Nepal's failed development was:

[...] not about the technical aspects of whether and how development has failed. It is about why is it that the country's precarious social and economic condition embedded in an uncertain and probably unhelpful external milieu does not attract commensurate behavioural response from the responsible quarters. The latter includes not only the state institutions and political parties but also the various agents and institutions of what is called the civil society.

He further writes (1999:2): "If there is development, the average citizen of the country should feel reassured that they are in greater control over their environment and that they have a command over the range of choices available to them for their future progress." While acknowledging that the academic definition of development can be disputed, he argued that (Panday, 1999: 7): "[...] the ordinary people recognise it when they feel it and enjoy it." Improved air quality or happier children are both conditions that people will notice immediately.

According to the World Health Organization (WHO 1999) and United Nations Development Programme (UNDP 2001), human poverty, poor health, food scarcity, and malnutrition represent major obstacles to social and economic development. Therefore, initiatives that are aimed at battling the causes of poor health are very important. In order to make such initiatives effective and not waste money and human resources we must distinguish between conditions that are given by nature and conditions that are created by people. It is impossible for people to stop earthquakes and volcanic eruptions, and they cannot change the harsh conditions for growing crops in high altitudes and in desert areas. Besides some technological solutions, for example, irrigation in desert areas, the only way to ease the living conditions is to migrate. But man himself has also contributed to poor living conditions. Heating with coal, exhaust from cars, industrial waste, etc., have created air and soil pollution that have 
affected people's health. At the individual level we know that cigarette smoking, excessive alcohol consumption, drug use, unsafe sexual relationships, and unbalanced nutrition create different types of illness.

\section{An Introduction of Communication Theory}

Communication is a process in which a sender transmits a message (ideas, facts, information) to a receiver. Communication can take place directly from person to person, or it can happen through mass media. During the 1930s and 1940s, even the mass media communication process was believed to be a direct one-way process from the sender to the receiver. This was called the Stimulus Response (S - R) model of communication. In an election study in 1940 (Lazarsfeld et. al., 1948), the researchers discovered that there was one or several opinion leaders who served as mediators of a sender's message to the receivers. They were so called 'gate keepers' who selected information in the mass media (the sender) and transmitted it to the audience. The two-step flow model had been created. It also turned out that people did not use just seek one person for information and judgements, but that they had different opinion leaders for different topics. Thus, they talked with one particular person about local affairs, with another one about fashions, and still another one when they wanted to know more about foreign affairs.

The lesson to be learned from this research is that we have to decide who our audience comprise, i.e. who the receivers of the message should be. Do we want to reach a mass audience, for instance, the inhabitants in a whole country, within a particular region, or in local areas? Or do we want to reach people with particular needs, that is, specific groups? Our choice of communication medium depends on this decision. The choice of mediating form also depends on what we want to transmit (the message), and what our purpose for initiating a communication process is.

Let us first look at who we want to reach. It can be a target population and/or a receiver group (Windahl and Signitzer, 1993). A target population comprises those individuals whose behaviour, attitudes or knowledge we want to influence, directly or indirectly, while a receiver group is the group for which a certain message is intended. We can look at these two audience types in three ways. First, the target population and the receiver group can be identical. For instance, we may want to reach all members of an ethnic group with a specific message with the intention of influencing their level of knowledge, their attitudes or their behaviour. Second, the receiver group can be situated within the target population. For instance, we may want to reach all women (target population) within a particular ethnic group (receiver group). Third, the receiver group can be situated outside the target population. For instance, we may want to reach professional health workers (the receiver group) who we hope can convey health information to a particular ethnic group or to women within a particular ethnic group (the target population).

This description of audience types has also alluded to what the purpose of a communication can be. People who initiate a communication process, be its parents, teachers, politicians, business managers, etc., want to make a difference by increasing people's awareness of a certain issue, to change their level of knowledge about various aspects of the issue, to influence people's values and attitudes that are related to the issue, and, finally, to change people's behaviour with respect to a specific issue. Awareness, knowledge, values and behaviour can be seen as stepping stones in a communication ladder. Thus, we cannot expect people to stop smoking if they are not aware of, or do not know about, the health risks 
involved. Certain values may represent an even more important obstacle to behavioural change. In this article we are particularly concerned with those values and attitudes that serve as significant cultural preconditions for successfully reaching specific target populations.

Any communication process includes a sender and a receiver who are sharing a message. There are several ways to look at what is going on between the two partners in this process. We have already described the traditional effect model in which a message was thought to be transmitted directly from a sender to a receiver. The two-step flow and multi-step flow introduced the diffusion model. Diffusion is a process by which an innovation is communicated through certain channels over time among members of a social system, this being a professional or occupational group, an ethnic or age group, etc. (Rogers, 2000). An innovation can be an idea (e.g. reasons for a particular illness), a practice (e.g. stopping smoking to prevent lung cancer), or an object (e.g. X-ray machine to look at parts of the body) that is perceived as new by an individual or by several people who have a common characteristic. A successful diffusion process begins when people get to know about a new idea, a new practice or a new object. Then they have to be persuaded that the new thing is good before they decide to adopt the new idea, practice or object. The final stage is when they actually have accepted the new idea or implemented a new practice or object, and that they after a while are being confirmed in their decisions.

The validity of the diffusion model was first tested within the agricultural sector. Research found that a few particular people collected information about, and then introduced in their farming, certain machines, seeds, fertilizers, etc. These people were called the early innovators, and it could take several months or years before knowledge and technology had been diffused to most of the farmers within an area.
However, once the tide turned, the curve for the number of people who had adopted the new technology became very steep. When the spreading of the innovation had lasted a while (months or years) the diffusion curve flattened.

Both the effect model and the diffusion model look at the communication process as going from one or more senders to one or more receivers, to an audience. The receiver does, however, consider how relevant the message/content is for himself/herself and selects accordingly. The discovery of the opinion leaders in the two-step flow communication process and of the early adopters in the diffusion process proved that such considerations took place.

The participatory model offers a totally different view of the relationship between those involved in a communication process. The participatory approach can be described as the opening of a dialogue in which sender and receiver interact continuously and reflect constructively about the situation, defining what their needs and problems are, and jointly discussing how needs can be met and problems can be solved, and finally, that the partners involved can actually begin to act accordingly. Participatory communication can be seen as an ideal speech situation between equal partners. It has also been called network communication. This implies that the distinction between sender, message and receiver becomes less relevant because there is an ongoing interaction in which people share relevant information in order to reach an agreement about what the problems to be solved are, and how to do it. With Panday's (1992: 2) words:”If there is development, the average citizens of the country should feel reassured that they are in greater control over their environment and that they have a command over the range of choices available to them for their future progress." 
Participatory communication is not easy to practice. It is a time consuming process which demands patience from all people involved. The figure below illustrates the complexity of the participatory model.

Figure 1. Information exchange and collective action in the participatory model

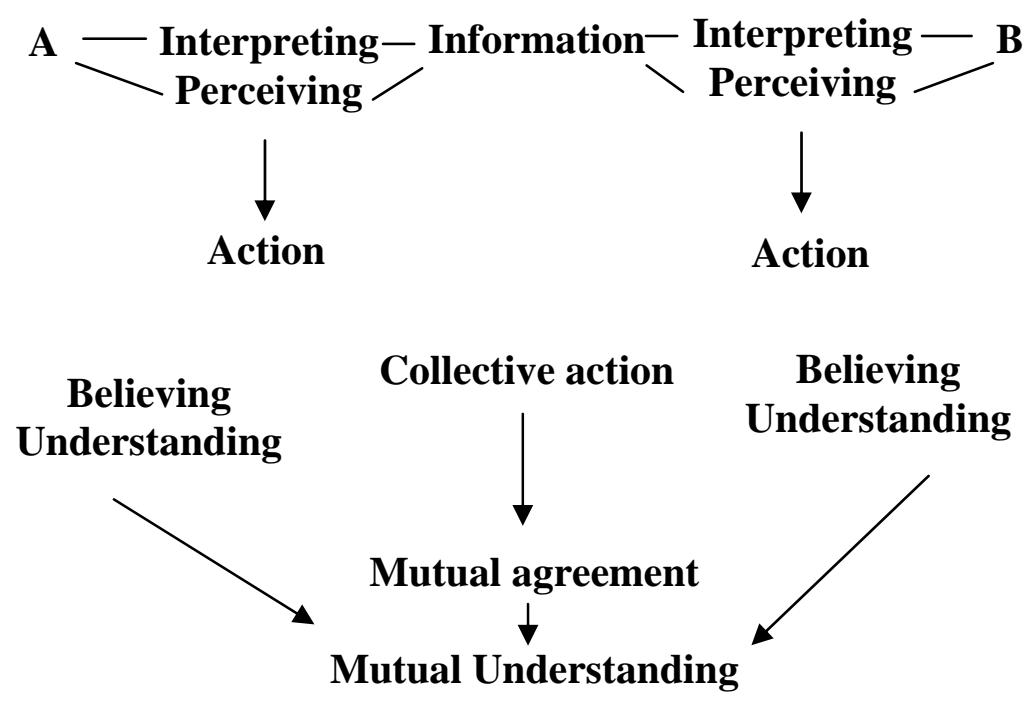

\section{Mutual understanding}

Let us use people's well being and health as an issue. In the figure above, A would be health workers such as nurses, medical doctors, practitioners in alternative medicine, nutrition, etc., while B can be people who live within a specific geographic area (a village, a region, etc) or who belong to a particular social group (e.g. women, ethnic group, etc). A basic prerequisite for $\mathrm{A}$ and $\mathrm{B}$ to have an interest in each other at all is that they see it worth while to exchange information. But what type of information? For instance, the
World Health Organization (WHO) publishes an annual report about the health conditions among the inhabitants of countries throughout the world. One example in Nepal is the disability survey that Impact Nepal (1998) did in the Sindhuli District. The survey registered the frequency of the following disabilities: hearing impairment, no speech and language, visual impairment, mentally retarded and physical disability. The percentage of disabled persons in the whole population in the Sindhuli District was 9 per cent (Impact Nepal, 1998: 6 ). Other studies have revealed a high degree of malnutrition which is the cause of several types of illness.

Do people themselves perceive the same health problems as the professionals? Most mothers notice quickly when a child has diarrhoea, but they do not always know the best way to treat it. It can be more difficult to discover - and to acknowledge - that a child is mentally disabled, and almost impossible to know that this can be caused by iodine deficiency. The relationship between $\mathrm{A}$ and $\mathrm{B}$ raises two issues. People concerned (B) may not be able to interpret correctly the symptoms that professionals (A) are trained to spot and understand. Equally important is the fact that mothers may perceive symptoms and illness as caused by fate (karma) and/or evil spirits. Thus, the information that A and $\mathrm{B}$ can exchange is obtained within different cultural contexts. This means that the very same facts are interpreted and perceived differently by A and B respectively. Therefore, we have to be prepared to spend much time on finding out what people (B) regard as problematic for their well being. It is not sufficient to approach mothers with a rational explanation that iodine deficiency causes mental disability.

To initiate some kinds of action may be one possible step forward. This was tried in Bhaktapur by a team of Norwegian doctors. Research has shown that zinc supplement can treat diarrhoea (Aase, 2002). Eventually the medical team 
managed to persuade mothers in the area to come regularly to the clinic so the child could be given a drop of zinc. It took not long before the number of children with diarrhoea decreased. Action had proved that there was some truth in what the medical team had told them. By inviting the mothers to come to the clinic, they met other women and could see that they had a common problem. The Norwegian team trained Nepalese health workers who, it was hoped, could inspire the women for collective action in the neighbourhood, that is, to continue with the zinc supplement if the diarrhoea returned. Eventually the women would understand the relationship between zinc and the absence of an illness, and thus believe in the importance of giving their children this particular supplement. By participating actively in this process health workers and indigenous women had reached a mutual agreement and a mutual understanding. The challenge in such initiatives is to make the behavioural change last over a longer period of time.

There are four factors that can influence people's willingness to enter into a participatory communication process. First, they must believe that the facts they are presented are true. In other words, when the mothers saw that a repeated drop of zinc healed their children's diarrhoea, the chances of them accepting this relationship as true increased. Second, people must see the relevance of a new idea or technique. This was probably the most important reason why the medical team managed to persuade women in Bhaktapur to come to the clinic in the first place; their children's health was at stake. Third, communication initiatives must be perceived as being sincere and serving the interest of people. Development aid and medical research can be problematic in this respect. Time-limited projects, be it in charge of national or foreign agencies, are done with good intentions. At the same time we must acknowledge that also professional prestige can be involved, for instance in a medical research project.
However, the crucial aspect of any innovation process is to transfer knowledge to the people who live in a neighbourhood, in a village or in a region. Again with Panday's (1999: 3) words:

Admittedly, there are instances of genuine accomplishments due to the enterprising efforts of some individuals or groups. But we cannot accept them as symbolic of "development", if they do not have the property of being a part of a cumulative, inter-linked, sustained, and socially inclusive process.

Thus, "good intentions" should not mean just doing something for people, but rather that people are given resources (knowledge, techniques, equipment, etc.) that can enable them to be in charge themselves of improving their living conditions.

The fourth factor that can make people trust a participatory communication process is comprehensibility. People must understand what is going on; that an innovation has implications for whatever their problems may be, and that they understand how to use a new technology.

At the individual or group level we must be aware of the character of the social relationship between those who know (A) and those who do not know (B). Between A and B two types of information are exchanged; content information and relationship information (Kreps, 1990). Content information includes facts, for instance, about the nature of health, reasons for different illnesses, and health care. On the other hand, relationship information conveys the level of concern, sensitivity, and power that health workers express toward patients and that health educators show during meetings with people (their audience). To be aware of this distinction is crucial for a participatory communication process to succeed. Health workers can be professionally excellent and score 
high on content information, but they fail dismally to get people involved because their relationship information indicate that people (e.g. women in a village) are not regarded as equal partners when defining what the problem may be as well as deciding the best way to solve it.

In order to understand the communication process between health workers (A) and people (B) we must know something about the cultural preconditions that each part brings into the relationship. Culture implies health beliefs with respect to four questions (Subedi 2001): (1) Why does this happen? (2) Why does this happen to me? (3) What can or should be done? (4) Who knows, and who can be trusted?

The first question addresses the causes for an illness. On the one hand, rational school medicine looks for physical, social or material circumstances. Many people have the same opinion about the reasons for good health, but we also know that beliefs in fate (karma) and evil spirits still exist. Such attitudes must be treated seriously in a participatory communication process. Culture can also offer explanations to why just a particular person or a particular social group is afflicted. An evil spirit aims at one person in particular, while zinc deficiency is a general phenomenon among children in many parts of Nepal. The cultural difference is the one between a personality oriented explanation, and a rational nutrition oriented explanation. Depending on the cultural beliefs, there are also different answers to the third question. An explanation based on fate or evil spirits asks for supernatural treatment, while the rational explanation implies treatment according to the principles of school medicine. This naturally leads to two different choices regarding whom to turn to for treatment; the shaman, the healer, or the medical doctor (e.g. Subedi, 2003).
Access is a prerequisite for development communication that governs people's participation in programs for social change. Availability of mass media in a country or specific community, however, does not guarantee that media will be used by the people. Multi-channel approach for development communication would ensure wider reach with lasting effect.

\section{Communication and Social Change}

The previous sections in this article should have made it clear that the purpose of initiating a communication process is to obtain changes in ideas, knowledge, attitudes, and behaviour. When the business community spends much money on advertising the goal is to persuade more people to buy certain products. Another example is when private and government agencies want to allure tourists by printing catalogues and paying for advertisements in national and international mass media. Also voluntary associations depend on communication to reach members with information and to recruit new members, and teachers communicate with their students. In other words, communication is an integrated part of social life.

Our concern in this article is how communication can be used to cope with social problems, and to focus on Nepal in particular. The discussions in the previous sections have made us aware of the distinction between objective and subjective definitions of social problems. We must also keep in mind that there may be different conceptions of social change and development, as expressed by Mishra (1987:105):

Development is somehow holy, uplifting and attractive. It is, however, also mysterious. The object is subjectively perceived and the totality of subjectivities does not add up to an objective description and/or assessment. 
We have also argued for a participatory communication approach in which people's subjective views are crucial. This does not mean, however, that objective data and indexes should be ignored. This type of statistics actually enables us to compare across countries and to identify the particular social problems in the country we are interested in. The participatory element becomes relevant once we reach the stage when we are ready to approach a special group of people.

What are, then, the social problems in Nepal? For several years the United National Development Programme (UNDP) has published an annual report about the state of affairs regarding human development in about 170 countries in the world. The data in these reports offer information about human well-being, not just economic trends, and form the basis for calculating a Human Development Index (HDI). The three basic dimensions in this index are (UNDP, 2001: 240):

- A long and healthy life, as measured by life expectancy at birth.

- Knowledge, as measured by the adult literacy rate and the combined primary, secondary and tertiary gross enrolment ratio.

- A decent standard of living, as measured by Gross National Product (GNP) per capita.

Depending on how countries score on this index, they are classified as having high, medium, or low human development. According to the 2001 index (UNDP 2001: 159), Nepal came fifth among 34 low human development countries, and 144th among a total of 174 countries. Nepal's HDI was 0.474 compared to Norway's score of 0.934 (the second highest in the world). The difference between Nepal and Norway can be illustrated by a few facts. For instance, in 2000 (UNDP, 2000: 169), it was expected that 21.9 per cent of the Nepalese population would not survive to age 40, while 8.9 per cent of the Norwegian population was expected to live until the age of 60 . The same report told us that 29 per cent of people in Nepal were without access to safe water, 90 per cent were without access to health services, and 84 per cent were without access to sanitation. The same facilities were available to the whole Norwegian population, and therefore not recorded in the statistical survey.

It is beyond any doubt that health workers and health administrators have a challenging job. Although this article is focusing on health and communication, it is worth repeating that not all social problems are communication problems. For instance, in the introduction in this article we quoted Brundtland (1999: viii) who emphasised the close relationship between poor health and poverty. There are, however, many reasons why people are poor. For instance, natural factors can cause harsh environments for growing crops in high altitude areas in Nepal, or they can create droughts in desert areas in Africa. We must also mention that people can be poor because the distribution of resources, including income, is unequal. This is a political matter that requires that citizens mobilize in order to influence political decisions that can change their life situation. Communication is a crucial tool for political mobilization, but this article will not delve any further into this. We shall move on to look at other reasons for poor health and particular diseases, and to what extent communication can contribute to solving health problems.

This is not the place for describing strictly medical explanations for poor health. Although they are important for health workers' choice of treatment, we are more interested in general factors that can cause diseases. In addition to poverty, illness can be caused by malnutrition (Aase, 2002; Andersen, 2002), infections (e.g. from the malaria insect), lifestyle (e.g. smoking, overuse of alcohol, fat food), poor 
sanitation, and polluted environment. These conditions can be attacked at a structural and/or at an individual level. At the structural level politicians can decide to establish welfare programs that can reduce poverty. Malnutrition can be reduced with programs for food supplements, and malaria can be fought by distributing bed-nets and medicine, and also by cultivating malaria rich marshes. Restrictions on advertising from the tobacco industry and information about the health risks related to smoking may change people's behaviour. Regulations regarding the use of private and public vehicles and industries in cities can improve air quality which, in turn, reduces the amount of respiratory problems. At the individual level education is the key to coping with all these health problems - education and some money.

Thus health problems can be approached by looking at health-directed factors (medical) and/or factors in people's life situation that are deemed to be health-related in the sense that they affect people's well-being in one way or another. Available health facilities, material infrastructure such as clean water, sanitation, pollution, etc., and health information are all health-related factors. The Ottawa Charter (1986: 1) defined health promotion in the following way:

Health promotion is the process of enabling people to increase control over, and to improve, their health. To reach a state of complete physical, mental and social well-being, andindividual or group must be able to identify and to realize aspirations, to satisfy needs, and change or cope with the environment. Health is, therefore, seen as a resource for everyday life, not the objective of living. Health is a positive concept emphasizing social and personal resources, as well as physical capacities. Therefore, health promotion is not just the responsibility of the health sector, but goes beyond healthy life-styles to wellbeing.
The Ottawa Charter listed five means to promote action for improved health:

- Building healthy public policy

- Creating supportive environments

- Strengthening community action

- Reorienting health services

- Developing personal skills and self-efficacy, i.e. people's judgement about their own ability to enact recommended behaviour

These quotes from the Ottawa Charter give us a short version of the importance of getting people involved in projects aimed at improving their social conditions, health included. We must also discuss to what extent changes in the type of structural elements listed in the Ottawa Charter are needed. For instance, to persuade the government in a country to invest in health facilities may require pressure from international organizations such as WHO and UNDP. It would also be beneficial for this case if the politicians acknowledged that they have something to gain politically by spending money on health provisions (Justice, 1986).

Over time there has been published many guides and handbooks about how to plan and implement a strategy for health communication (e.g. Haaland, 2001). The Center for Communication Programs at Johns Hopkins School of Public Health in the United States is regarded as one of the top institutions for testing health communication material. In their book Health Communication, Phyllis T. Piotrow and colleagues (1997) focused on family planning and reproductive health. This is, of course, a crucial issue in health policy and practical work, but the book is also relevant for other aspects of people's health. The book combines theoretical reflections with descriptions of how different types of communication material have been tested and evaluated. The book describes five steps for designing a 
strategy for health communication that is aimed at enhancing social development: (1) Setting objectives; (2) Positioning; (3) Assessing communication strategies; (4) Assessing implementing organizations; (5) Documentation and evaluation.

\section{Setting objectives}

It is important to spend some time on the first step. The previous section showed that successful communication requires that we must have decided who we want to reach with a specific message. We have also seen that the 'who' in the communication process can be people who define health policy objectives at the national level, in specific areas, or among specific population groups. Programs to accomplish our goals depend on who we want to reach. Piotrow et.al. (ibid.: 59) mention "high quality of health care, more integrated reproductive health services, or economic sustainability over time". The time element is important (ibid.: 60): "Long-term vision can set bold objectives. [...] Every program needs a long-term vision. It can empower people because it shows what is important. It can stimulate teamwork because it shows what everyone needs to do. And it can strengthen organization because it generates new energy." So many programs have failed because public health officials or political leaders want quick results without having to spend too many resources.

Communication objectives should be SMART (ibid.: 63):

S Specific - defining what is to be accomplished in terms of specific steps to behavioural change among specific, well-defined audiences

M Measurable - quantifying the objectives by indicating a numerical or percentage change expected

A Appropriate - defining intended changes that are culturally and locally acceptable
R Realistic - avoiding objectives that are beyond the scope of available resources, contrary to relevant experience, or unrelated to communication efforts

T Time bound - identifying the time frame in which changes should be achieved

It may be difficult to measure in numerical terms the changes that we want to obtain. However, some kind of change should be defined before we start planning a time consuming communication process. For instance, we should know whether we want to reach the whole population - that is, a mass audience - or specific groups, either as receiver group or target group as described in section 4 .

\section{Positioning}

The term positioning comes from the commercial world and its strategic implication is to present (Piotrow, 1997: 64) “[...] an issue, service, or product in such a way that it stands out from other comparable or competing issues, services, or products and is appealing and persuasive.” The point with positioning is that people should become aware of, and understand, the purpose of a particular program. This can be obtained by the use of a certain terminology or images. In countries with high illiteracy rate images have proved to be a very effective way to create awareness about health issues (Haaland, 2001). Positioning must take cultural values into consideration. Piotrow et.al., (1997: 67) illustrates this by describing many Western nations' emphasis on individual human rights in contrast to the Chinese political system's focus on national interests. For instance, political leaders in China have promoted family planning as a patriotic duty. The answers to health related questions also depend on people's 
cultural context (Subedi, 2001). This aspect of culture must be taken into consideration also in the positioning phase.

\section{Assessing communication strategies}

To assess communication strategies requires a discussion of which steps to take at different phases. We cannot expect anything but failure if we tell mothers that zinc supplement is good for their children before a system for making the supplement available has been established. Thus, the clinic in Bhaktapur was set up with a medical team and a training program for Nepalese health workers before they told people in the neighbourhood about the program. It can also be worth while to consider whether to launch a nation-wide campaign or to begin communicating to small groups.

The PABASA program in the Philippines (Solon, 1999) is an excellent example of a communication design that begins with a small group and expands to whole villages. First, a team of nutrition experts from the Nutrition Center of the Philippines (NCP) got permission from the village chairperson to invite about ten women - who were seen as opinion leaders - to a two day meeting to learn about nutrition, healthy food, sanitation, etc. These women afterwards formed other groups, and the process continued until, if all went well, the new information had reached all households in the village. The PABASA program also illustrates a multimedia approach by using informal discussions, games, brochures, posters, and information about nutrition given by the local store keeper. Campaigns in mass media such as television and radio can be effective to raise awareness among a large audience, but other mediating forms seem to be better means if we also want behavioural change.
We often perceive health information as very serious. The content in health communication is indeed serious, but serious information can be presented in an entertaining way. Research has shown (Piotrow et.al., 1997: 76; Storey, 1999) that popular songs, television series, soap operas, and theatres performances on the village square increase people's curiosity about health issues. Apparently it is also easier to remember facts that have been presented in an entertaining form and not as plain facts. This is particularly important when our audience are young people. Research (e.g. Green, 2002) has shown that hard facts about health related behaviour can be ignored by adolescents because they are risk takers. They simply do not believe that "this can happen to me", and besides, it is exciting to challenge their luck.

Finally, communication strategies should be designed in such a way that the information is given by credible sources (people and/or institutions) that are trusted and respected by the audience we want to reach (Piotrow et.al., 1997: 81).

\section{Assessing implementing organizations}

Our next step is to make a plan for implementing the communication strategy we have designed. Who will be doing it, and how to do it, are the two crucial issues at this stage. Health communication requires resources that organizations, and not individuals, can provide. Piotrow et.al. (1999: 84) write that "The major criteria for implementing organizations are competence, commitment, clout, and continuity."

The projects that we have described from Bhaktapur and the Philippines (Solon, 1999) were run by organizations that sent people with a professional training into the field. The 
PABASA project in particular illustrates a participatory approach in which women in local communities were treated as equal partners in bringing nutrition and health information throughout the village. Community participation is often crucial for health communication to succeed (Cohen,1996).

There is one problem with using professionals in health communication. In section 4 we distinguished between content information and relationship information (Kreps, 1990). Aubel and Niang (1996) made a study of midwives' interpersonal communication behaviour during family planning consultations in Senegal. They found that some of the consultations did not go well because the midwives had a top-down "I-know-best" attitude toward their clients. Thus the women did not fully receive or acknowledge the objectively correct content information because they perceived the relationship information negatively. The study's recommendation was (Aubel and Niang, 1996: 79):

From the beginning to the end of the family planning consultation the midwives should involve the client in a dialogue in which the client is encouraged to ask questions, and is asked her opinion in terms of decisions to be made and follow-up action to be taken.

\section{Documentation and evaluation}

The last, but not least important, step in Piotrow and colleagues communication strategy is documentation and evaluation. Everything we do from setting objectives to implementing a communication strategy must be documented. What are our goals, what type of change in awareness, knowledge, attitudes, and behaviour do we want to obtain? Which group of people do we want to reach with health information? How can we best communicate to our receiver and target groups? And how are we going to implement the communication strategy we have agreed upon? It is only such detailed documentation that enables us to evaluate if we have reached our goals, or if we are on the right track.

\section{Cltural Preconditions}

Although culture is a slippery concept which may be difficult to grasp, we nevertheless, cannot be without it. In a general sense, culture includes beliefs, values, feelings, symbols and meanings, foods, and modes of social interaction. Human beings orient themselves in space and time, by means of language and social relations, with the body, food and clothes, by the structure of everyday life, and in terms of symbols and framework provided by public myth, religion and rituals (Delaney, 2004). It is important to dig deeper and analyze both the particular meaning embodied in these phenomena and the way they are interconnected. If we are trying to give health education to a specific group of people with the purpose to change their existing practices, it is important to know their total way of life and how they perceive it. This helps us to understand them better and to assess the best way forward to change their behaviour. However, it is extremely important to investigate the ways in which power (aggression, repression, and exploitation) influences cultural expressions.

The following overview (Table No. 1) enables us to compare cultural expressions among women in five different ethnic 
groups in Nepal. It shows the terms used locally by the mothers

Table No. 1: Terms and Expression to Describe Pneumonia and Danger Symptoms

\begin{tabular}{|c|c|c|c|c|}
\hline Brahman/Chhetri & Tamang & Tharu & Musahar & Muslim \\
\hline $\begin{array}{l}\text { Swah-swah } \\
\text { garne/Chhito Chhito } \\
\text { swash ferne (fast } \\
\text { breathing) } \\
\text { Ghyar ghyar garne } \\
\text { (Noisy breathing) } \\
\text { Saas pherna garo } \\
\text { (difficulty in } \\
\text { breathing) } \\
\text { Kokha hanne (chest } \\
\text { in-drawing) } \\
\text { Salang sulung hune } \\
\text { (Weakness/Convulsi } \\
\text { on) } \\
\text { Sarir/nidhar tato } \\
\text { hune (increased } \\
\text { body temperature) } \\
\text { Nasutne (Unable to } \\
\text { sleep)/ Sutirahane } \\
\text { (too Sleepy) } \\
\text { Khana kam khane } \\
\text { (loss of appetite) } \\
\text { Dudh nakahane } \\
\text { (don't feel like } \\
\text { suckling) }\end{array}$ & $\begin{array}{l}\text { Kokha hanne } \\
\text { (Chest in- } \\
\text { drawing) } \\
\text { Ghyar ghyar } \\
\text { garne (Noisy } \\
\text { breathing) } \\
\text { Na nabha khaji } \\
\text { (unable to } \\
\text { cough out } \\
\text { mucous) } \\
\text { Ghanti sar sar } \\
\text { lazi (noisy } \\
\text { breathing) } \\
\text { Na khaji, } \\
\text { (mucous flow), } \\
\text { Nodpa } \\
\text { (Cough), Jar } \\
\text { khaji (fever) } \\
\text { Aankhami } \\
\text { (unable to eat) } \\
\text { Po boba } \\
\text { (swollen } \\
\text { stomach) } \\
\text { Simbatu khaji } \\
\text { (cold sweat) } \\
\text { Nepale/Mastira } \\
\text { Lagyo } \\
\text { (pneumonia), }\end{array}$ & \begin{tabular}{|l} 
Jar aune \\
(fever), \\
Khokee lagne \\
(Cough), \\
Swah Swah \\
garne (Difficult \\
breathing) \\
Kokha \\
dumaiche \\
(Chest pain) \\
Dulki Jar \\
(Shivering \\
fever), \\
Ghanti Ghar \\
Ghar Karchhe \\
(Noisy \\
breathing) \\
Dhekarwa \\
(Convulsions)
\end{tabular} & \begin{tabular}{|l|} 
Aankha \\
munne (Stare \\
for a long \\
time) \\
Naak sur sur \\
karche \\
(breathing \\
problem) \\
Ghanti ghar \\
ghar karche \\
(Noisy \\
breathing) \\
Neta ayeche \\
(Unable to \\
Cough out, \\
mucous) \\
Paanj \\
mariche \\
(Chest in \\
drawing) \\
Chatpat \\
garne \\
(restlessness), \\
Bachha \\
roiche (Cry \\
all the time) \\
Bhundi Tala \\
Mathi Jane \\
(Strong \\
movement of \\
Stomach) \\
Pajra \\
khapche \\
(Chest in- \\
drawing) \\
\end{tabular} & \begin{tabular}{|l} 
Jukam, \\
Thandi, \\
Bhukar \\
(cough, \\
Cold, \\
Fever) \\
Naak sur \\
sur karche \\
(breathing \\
problem) \\
Pajra \\
Marche/Kh \\
apche \\
(chest in \\
drawing) \\
Ma ke dudh \\
nakhaiche \\
(unable to \\
suckle) \\
Ultia \\
Aaiche(Vo \\
miting),
\end{tabular} \\
\hline
\end{tabular}

to describe acute respiratory infections (ARI) and illnesses among children. It seems very clear that mothers from different caste/ethnic background use different words (language) to indicate the similar type of sign and symptoms. In order to understand the case history of a sick child, it is a precondition to know the terms expressed by mothers in different caste/ethnic groups. To improve people's health condition it is necessary to explore the existing health seeking behaviours within and between the groups. Accessability, availability and accountability of the health facilities and health care practitioners play an important role for people's search among different health facilities and health care practitioners. Also the cost of such facilities is, of course, a very important factor for their choice of healers.

\section{Implication for the Communication Strategy}

Health communication encompasses the study and use of communication strategies to inform and influence individual and community decisions that enhance good health. Health communication can contribute to disease prevention and health promotion, and is relevant in a number of contexts, including patient-practitioner relations, individual's exposure to search for, and use of, health information, the construction of public health messages and campaigns, risk communication, images of health in the mass media, and the culture at large. It can also educate consumers about how to gain access to public health facilities, to know about different aspects of the health care system, including telehealth applications. The practice of health communication contributes to health promotion and disease prevention in several areas through training of health professionals and patients in effective communication skills.

For individuals, effective health communication can help raise awareness of health risks and solutions, provide the motivations and skills needed to reduce these risks, and help them find support from other people in similar situations. It can also increase demand for appropriate health services and 
decrease demand for inappropriate health services. It can make available information to assist in making complex choices, such as selecting health plans, care providers, and treatments. For the community, health communication can be used to influence the public agenda, advocate for policies and programs, promote positive changes in the socioeconomic and physical environment, improve the delivery of public health and health care services, and encourage social norms that benefit health and quality of life.

Another area is the dissemination of health messages through public education campaigns that seek to change the social climate to encourage healthy behaviours, create awareness, change attitudes and facilitate individuals to adopt prescribed behaviours.

In order to focus on key health improvement activities, effective health communication is a necessary precondition. The promotion of regular physical activity (physiotherapy or yoga), reading and sleeping habits, healthy and nutritional food, healthy weight, and safe and responsible sexual behaviour require a range of information, education and advocacy efforts.

Health communication alone, however, cannot change systemic problems related to health, such as poverty, environmental degradation, or lack of access to health care services, but comprehensive health communication programs should include a systemic exploration of all the factors that contribute to health and the strategies that could be used to influence these factors. Well designed health communication activities help individuals better understand their own and their communities' needs so that they can take appropriate actions to maximize health.

\section{Reference}

Aase, Tor Halfdan (2002). "Micronutrients and Ethnicity of diet in Arun Valley.” In Ram P. Chaudhary et. al. (eds) (2002) Vegetation and Society. Their Interaction in the Himalayas, pp. 137-148. Kathmandu: Tribhuvan University, and Bergen: University of Bergen. Andersen, Peter (2002). "Geographical Approaches to Micronutrient Deficiencies in Himalaya.” In Ram P. Chaudhary et. al. (eds) (2002) Vegetation and Society. Their Interaction in the Himalayas, pp. 137148. Kathmandu: Tribhuvan University, and Bergen: University of Bergen.

Aubel, Judi and Aminata Niang (1991). "Health workers' attitudes can create communication barriers." World Health Forum, Vol. 12, pp. 466-471.

Brundtland, Gro Harlem (1999). "Message for the Director-General." In World Health Organization (1999) Making a Difference. The World Health Report. Geneva: WHO.

Cohen, Sylvie I. (1996). ”Mobilizing Communities for Participation and Empowerment” . In: Servaes, Jan et. al. (eds) (1996) Participatory Communication for Social Change, Chapter 13. New Dehli/Tousand Oaks/London: Sage Publications.

Delaney, Carol (2004). Investigating Culture: An Experiential Introduction to Anthropology. United Kingdom: Blackwell Publishing.

Freire, Paulo (1972). Pedagogy of the Opressed. Harmondsworth: Penguins.

Greene, Kathryn et.al. (2002). ”Elaboration in Processing Adolescent Health Messages: The Impact of Egocentrism and Sensation Seeking on Message Processing." Journal of Communication, Vol. 52, no. 4 pp. 812-831.

Haaland, Ane el.a. (2001). Reporting with Pictures. A concept paper for researchers and health policy decicion-makers. Geneva: UNDP/World Bank/WHO.

Helman, Cecil (1990). "Cultural factors in health and illness." In: McAvoy, Brian R. and Liam J. Donaldson (eds) Health Care for Asians, pp. 17-27. Oxford/New York/Tokyo: Oxford University Press.

HMG-N/NPC (2002). Tenth Plan (2002-2007). Kathmandu: His Majesty's Government, National Planning Commission. 


\section{Dhaulagiri Journal of Sociology and Anthropology Vol.2 | 99}

Impact Nepal (1998). Disability Survey in Sindhuli District, Nepal. Report. Kathmandu: Impact Nepal.

Justice, Judith (1986). Policies, Plans \& People. Culture and Health Development in Nepal. Berkeley/Los Angeles/London: University of California Press.

Kreps, Gary L. (1990). “Communication and Health Education.” In Eileen Berlin Ray and Lewis Donohew (eds) Communication and Health Systems and Applications.. Hillsdale, NJ: Lawrence Erlbaum Associates Publishers.

Lazarsfeld, Paul et. al. (1948) [1944]. The People's Choice. How the Voter makes up his Mind in a Presidential Campaign. New York: Columbia University Press.

Melkote, Srinivas R. and H. Leslie Steeves (2001). Communication for Development in the Third World. Theory and Practice for Empowerment. London: Sage.

Martinez, Esperanza and Hari Koirala (2002). Primary Health Care Services in Nepal. Kathmandu: USAID.

Mishra, Chaitanya (1987). Development and Underdevelopment: A Preliminary Sociological Perspective (105-35). Occasional Papers in Sociology and Anthropology, Vol. I. Kathmandu, Central Department of Sociology and Anthropology, Tribhuvan University.

MoH (2004). Vulnerable Community Development Plan for Nepal Health Sector Program Implementation Plan. Kathmandu: Health Sector Reform Unit, Planning Division, Ministry of Health.

MoH (1999). Second Long Term Health Plan. Kathmandu: Ministry of Health.

NESAC (1998). Nepal Human Development Report 1998Kathmandu: NESAC

( Nepal South Asia Centre).

Ottawa charter for health promotion (1986). Report of the International Conference on Health Promotion. Ottawa, Ontario, Canada.

Panday, Devendra Raj (1999). Nepal's Failed Development. Reflections on the Mission and the Maladies. Kathmandu: Nepal South Asia Centre.

\section{Marit Bakke and Madhusudan Subedi}

Piotrow, Phyllis Tilson, et. al. (1997). Health Communication Lessons from Family Planning and Reproductive Health. Chapter 4 "Strategic Design." Westport, Connecticut \& London: Praeger.

Rogers, Everett M (1995 $4^{\text {th }}$ edition) [1962]. Diffusion of Innovations. New York: The Free Press.

Storey, J. Douglas (1999). "Popular Culture, Discourse, and Development: Rethinking Entertainment-Education From a Participatory Perspective." In T. Jacobsen \& J. Servaes (eds) Theoretical Approaches to Participatory Communication, pp. 337358. Cresskill, NJ: Hampton Press.

Subedi, Madhusudan Sharma (2001). Medical anthropology of Nepal. Kathmandu: Udaya Books.

Subedi, Madhusudan (2003). "Healer Choice in Medically Pluralistic Cultural settings: An Overview of Nepali Medical Pluralism." Occasional Papers in Sociology and Anthropology, Vol. VIII: 128158.

Subedi, Madhusudan (2004). "Indigenous Knowledge and Health Development in Nepal: an Anthropological Inquiry.” Manav Samaj, June 2004: 135-151.

Subedi, Madhusudan (2005). "Foreign Aid, Sustainable Development and Rapti IRDP.” Occasional Papers in Sociology and Anthropology, vol. 9, pp. 231-257. Kathmandu: Central Department of Sociology and Anthropology, Tribhuvan University.

UNDP (2000). Human Development Report 2000. New York/Oxford: Oxford University Press.

White, Kate (1999). "The Importance of Sensitivity to Culture in Development Work." In Thomas Jacobsen \& Jan Servaes (eds) Theoretical Approaches to Participatory

Communication, pp. 17-49. Cresskill, NJ: Hampton Press.

World Health Organization (1999). Making a Difference. The World Health Report. Geneva: WHO.

Windahl, Sven and Benno Signitzer (1993). Using Communication Theory. An Introduction to Planned Communication. London: Sage.

Woollard, Robert (2005). Feasibility Study for the Proposed Patan University of Health Sciences (PUHS). Kathmandu: Medical School Steering Committee. 